\title{
Studies on the Cytokinesis in Grasshopper Spermatocytes Treated with Hypotonic and Hypertonic Media
}

\author{
Ken-ya Kawamura
}

Biological Department, Faculty of Science, Tokyo Metropolitan University,

Setagaya-ku, Tokyo, Japan

Received April 27, 195\%

In cell division, although it is usually apparent that the position of the cleavage furrow is related to the position of the spindle body, the exact relationship of two is not yet clear.

In the present study the effects of hypotonic and hypertonic media on grasshopper spermatocytes were observed in order to analyse this relationship.

The writer wishes to express his sincere thanks to Dr. Katsuma Dan for his valuable advice and aid in the preparation of this manuscript.

\section{Material and methods}

The primary spermatocytes of grasshopper, Acrydium japonicum, were used throughout the experiments. Germ cells of the grasshopper were prepared into hanging-drop according, essentially, to the procedure previously described (Kawamura 1955): By carefully cutting the follicular walls with a sharp knife, spermatocytes with a small amount of body fluid were extracted and placed upon a cover slip. The cover slip was then inverted over a depression slide which was slightly moistened with water on the bottom and sealed with liquid paraffin. This procedure must be carried out swiftly and carefully.

The concentration of the culture medium was varied by changing the vapor pressure of the water in the depression by controlling the temperature of it (Fig. 1). By this means since the culture medium does not need to be

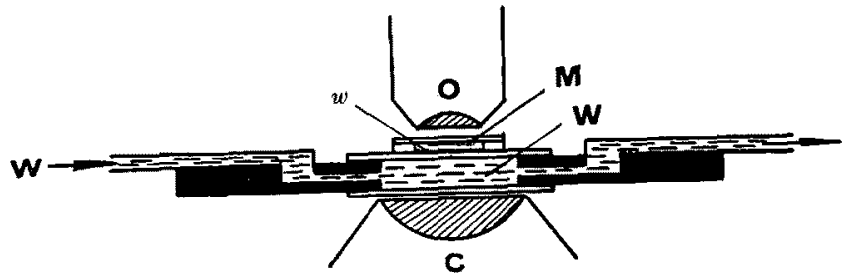

Fig. 1. Schema showing apparatus for varying concentration of culture medium. O, objective lens. M, materials. W, temperature controlled water. $\mathrm{w}$, a small amount of water. $C$, condenser lens. changed, the cells are not lost from microscopic view, and successive tracing of a single cell is easily accomplished through the process of changing the concentration of the medium.

Observations were carried out with the aid of a phase contrast micro-

1. Present address: Department of Zoology and Entomology, University of Tennessee, Knoxville, Tennessee, U. S. A. 
scope, using Zettnow's filter solution, periplan ocular $\times 10$ (Olympus), negative-medium contrast optics $\times 90$ (Tiyoda), at a temperature of approximately $23^{\circ} \mathrm{C}$.

The photomicrographs were taken with PM-6 (Olympus) by using Fuji $35 \mathrm{~mm}$ Neopan S film.

\section{Results}

Normal cytokinesis: A peculiarity in the mode of cytokinesis in Acrydium japonicum is that spindle poles are directly anchored to the surface layer of the cell (Kawamura 1957). During the telophase, the cell diameter along the spindle axis elongates strikingly, and a wide furrow is formed initially with a relatively long interzonal region. Later on, an incision is formed at the middle part of the interzonal region of the spindle (Pl. XIII Figs. 2 17). During telophase, the spindle length is increased to approximately 1.5 times that of the metaphase spindle length.

Cytokinesis in hypertonic medium: Hypertonicity was obtained by lowering the temperature of the water in the depression. Figures 18 29 show the successive stages of the cytokinesis of the primary spermatocyte in hypertonic medium. The spindle length in the telophase is approximately 2.4 times that of the metaphase spindle length. In spite of that, the cleavage furrow was formed almost normally.

Cytokinesis in hypotonic medium: Hypotonicity was obtained by raising the temperature of the depression to enhance evaporation from the water with depression which is deposited in the hanging-drop. Figures 30 44 show successive stages of cytokinesis of the primary spermatocyte in hypotonic medium. As shown in the figures, elongation of the spindle length during the telophase is suppressed and it is approximately 1.3 times that of the metaphase spindle length. The cleavage pattern is very unusual in which a furrow cuts a cell into round daughter cells, and is similar to that in the sea urchin egg.

Cytokinesis of the primary spermatocyte transformed from isotonic into hypotonic media at telophase: Figures 45 52 show successive changes of the dividing primary spermatocyte which was removed from isotonic into hypotonic media. When a normal cell at the late telophase is put into hypotonic medium, incipient daughter cells round up as if the cell surface is loosened from internal structures at both ends of the interzonal region. However, the surface of the equatorial plane remains dented in suggesting the surface still retains a connection with the spindle body. As shown in the figures, spindle length is decreased to approximately 0.7 times that in the normal telophase by this treatment. Nevertheless, the cleavage furrow incises the cell as the spindle length diminishes.

Cytokinesis of the primary spermatocyte with alternation between hyponicity and isotonicity: Figures 53 64 show successive changes of the 

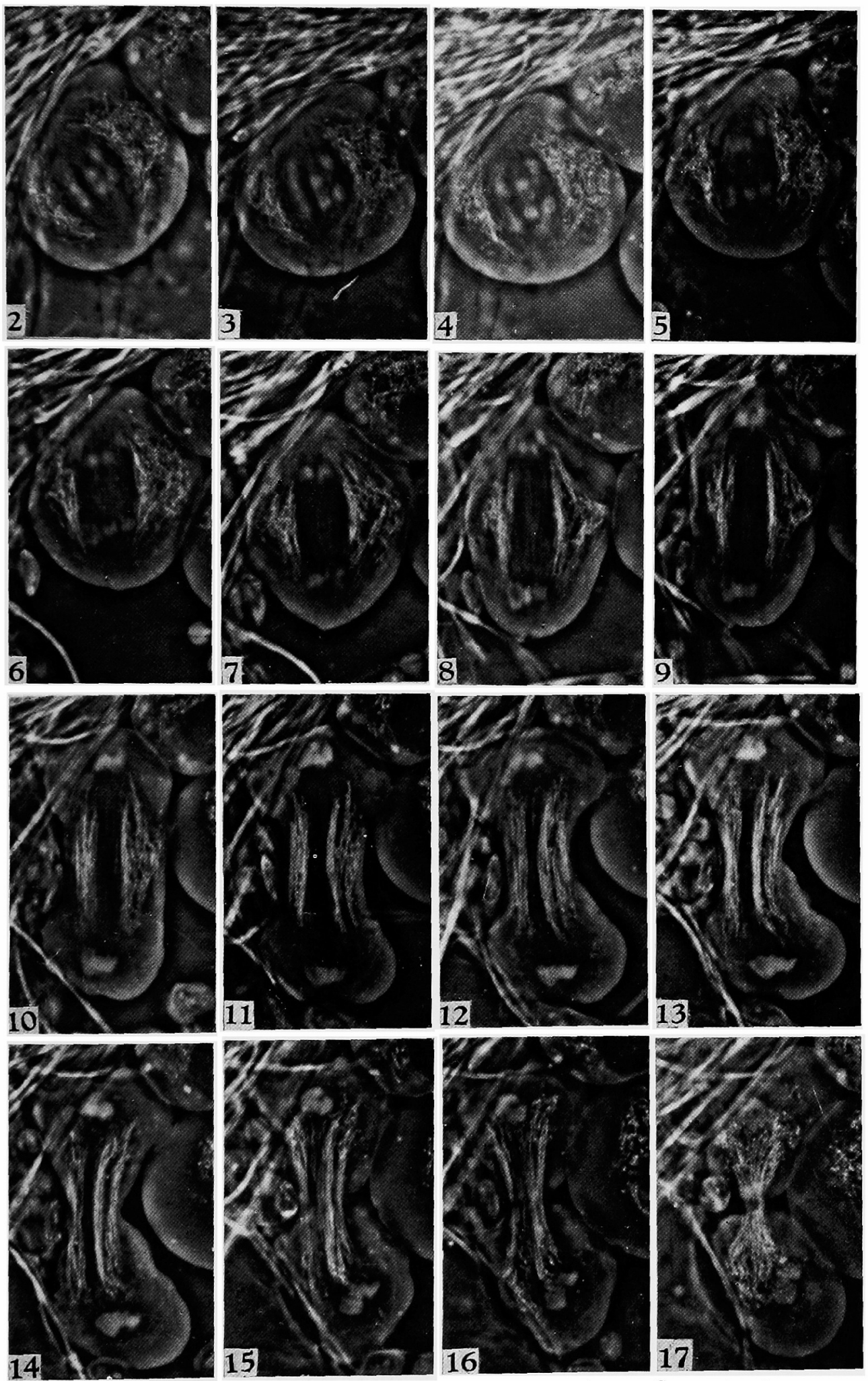

K. Kawamura: 

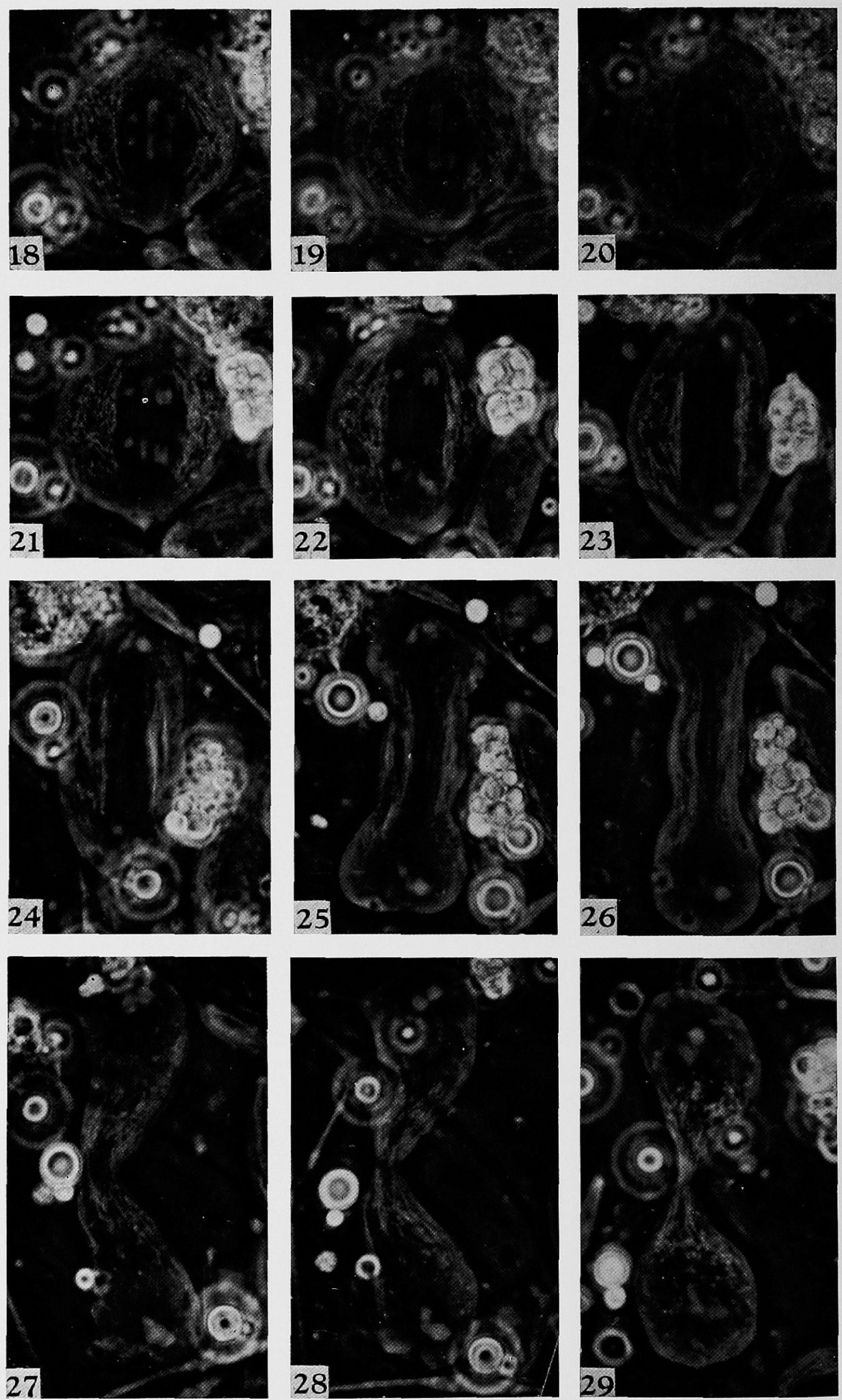

K. Kawamura: Studies on the Cytokinesis in Grasshopper Spermatocytes 

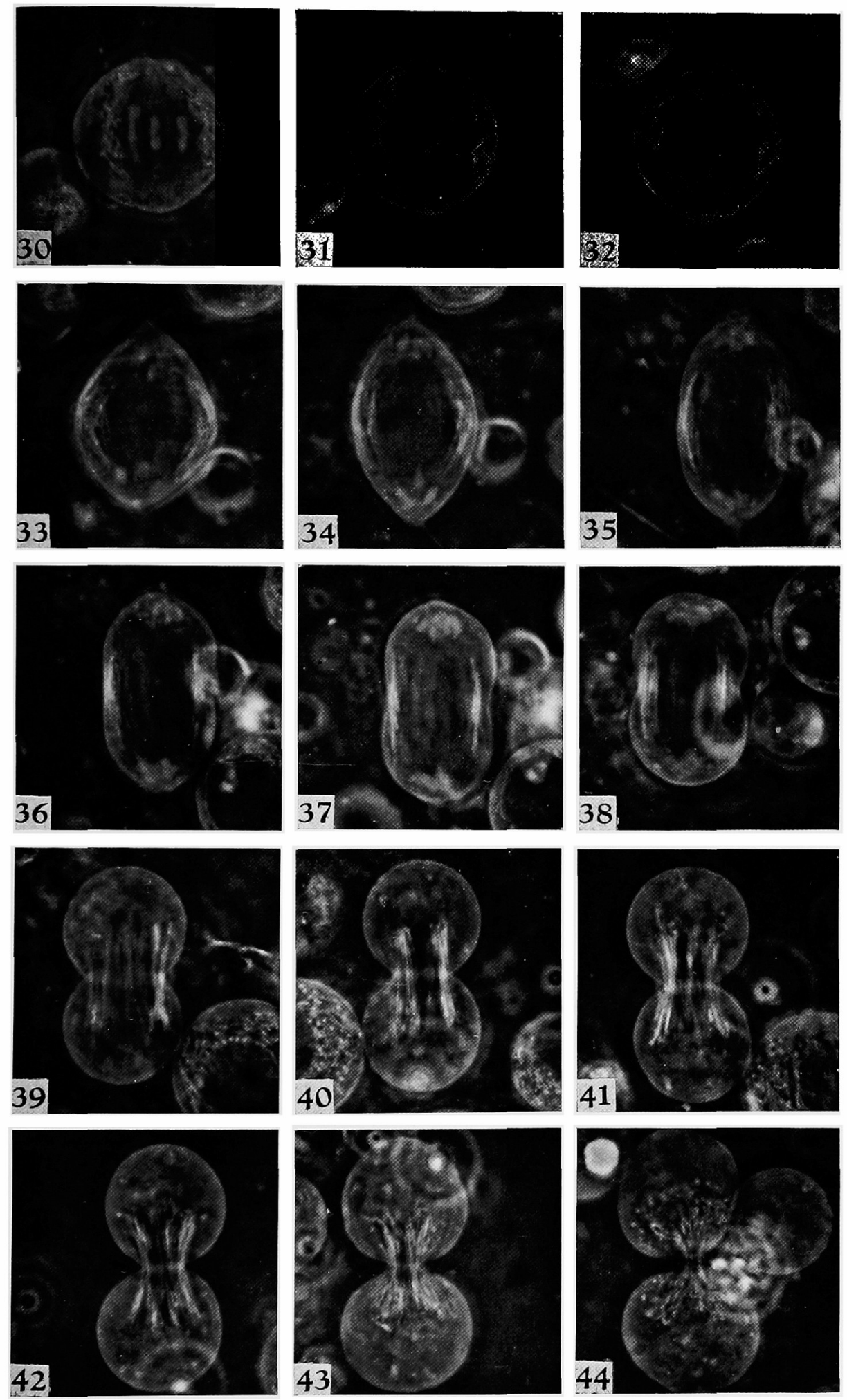

K. Kawamura: Studies on the Cytokinesis in Grasshopper Spermatocytes 

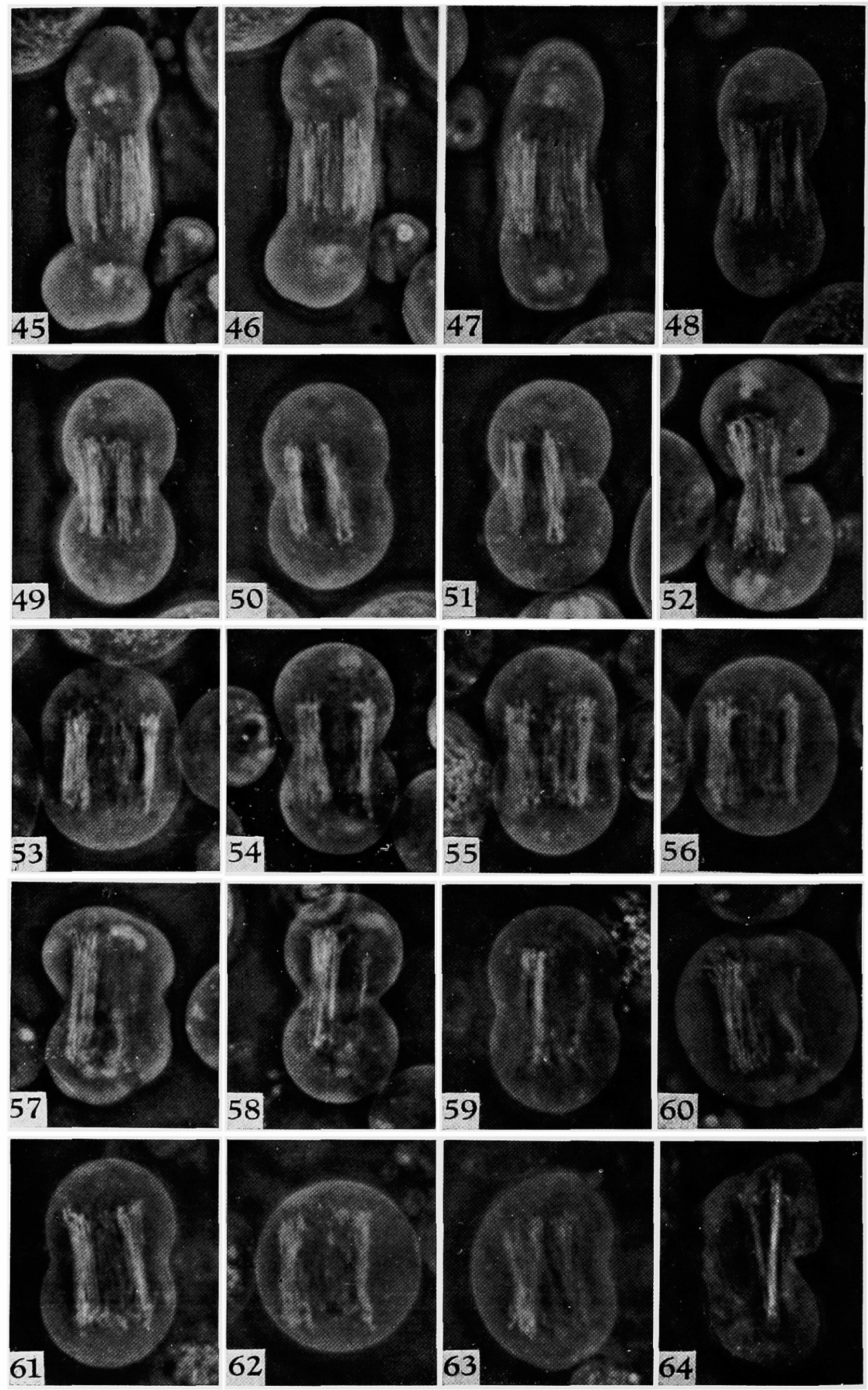

K. Kawamura: Studies on the Cytokinesis in Grasshopper Spermatocytes 
primary spermatocyte treated alternately with hypotonic and isotonic media. When cell in early telophase is put into the hypotonic medium, the cleavage furrow is completely reversed which reappears on a return to the isotonic medium. It is possible to repeat the operation several times.

\section{Remarks}

Although there are some publications by Shinke (1941) and Kanô (1951) concerning the effects of hypotonic and hypertonic solutions on dividing cells, those observations were carried out with primary regard to the abnormality of karyokinesis.

In the present study, the change of the spindle length is noteworthy. The spindle length of spermatocyte treated with hypertonic medium is elongated considerably, while by the treatment of hypotonic medium, elongation of the spindle was suppressed somewhat. Bèlar (1929) has also reported evidence of the elongation of the spindle body in hypertonic treatment in the spermatocyte of Chorthippus lineatus. Although the mechanism of the spindle elongation is still unknown, it is interesting that the length of the spindle body is controlled by the concentration of the culture medium. Furthermore, when a normal cell is placed in a hypotonic medium, the spindle length is decreased, although, the cleavage furrow incises the cell as the spindle length diminishes. Dan (1943) has assumed an autonomous elongation of the spindle body in his astral cleavage theory. Facts observed here indicate that elongation of the spindle body is not neccessary for the cleavage in grasshopper spermatocyte.

As stated above, the normal cytokinesis in Acrydium is carried out with a peculiar cleavage pattern in comparison with other grasshopper, but in the hypotonic condition, the pattern of cytokinesis becomes quite similar to other cases.

By alternate treatment of hypotonic and isotonic media, it is possible to repeat furrowing several times. When a normal cell at the late telophase is put into hypotonic medium, the incipient cells round up, but the central portion of furrow does not puff out from the spindle body.

Kuno (1954) has reported that there is a difference in the nature of the cleaving egg surface in sea urchin between the polar and the furrow region, and suggested that the egg surface or the cortex might play an important role in cleavage. Results obtained here suggest that the surface layer of the cell, in addition to the mitotic apparatus, might perform an important task in the cytokinesis of grasshopper spermatocyte. 


\section{Summary}

The effects of hypotonic and hypertonic media on the spermatocytes of the grasshopper, Acrydium japonicum, were observed with special reference to the cytokinesis and spindle.

Changes of the cleavage pattern and spindle length were described and discussed.

\section{Literature cited}

Bĕla r̆, K. 1929. Beiträge zur Kausalanalyse der Mitose. II. Untersuchugen an den Spermatocyten von Chorthippus lineatus Panz. Roux' Arch. Entw. 118: 359 484.

Dan, K. 1943. Behavior of the cell surface during cleavage. VI. On the mechanism of cell division. Jour. Fac. Sci., Tokyo Imp. Univ. 6: 323-368.

Kanô, K. 1951. Some observations of abnormal divisions in grasshopper germ cells after treatment with hypotonic and hypertonic solutions. Jour. Fac. Sci., Hokkaido Univ. 11: 139-149.

Kawamura, K. 1955. The course of spindle formation in the spermatocyte of the grasshopper, Acrydium japonicum, observed by phase microscopy. Cytologia 20: 4751.

- 1957. Studies on the mitotic apparatus and cytokinesis of spermatocytes of nine species of grasshopper. Cytologia 22: 337-346.

Kuno, M. 1954. On the nature of the egg surface during cleavage of the sea urchin egg. Embryologia 2: 33-41.

Shinke, N. 1941. The hydration and dehydration phenomena in mitosis. III. Apparent changes in structure of the nucleus. Cytologia 12:1-13.

\section{Explanation of Plates XIII-XVI \\ Plate XIII}

2-17. Sucessive series of normal cytokinesis in a single primary spermatocyte of Acry. dium japonicum $(\times 900)$.

\section{Plate XIV}

18-29. Successive series of cytokinesis of a primary spermatocyte in hypertonic medium $(\times 900)$.

\section{Plate XV}

30-44. Successive series of cytokinesis of a primary spermatocyte in hypotonic medium $(\times 900)$.

\section{Plate XVI}

45-52. Successive changes of a primary spermatocyte transformed from isotonic into hypotonic media $(\times 900)$. 53-64. Successive changes of a primary spermatocyte treated alternately with hypotonic and isotonic media $(\times 900)$. 